\title{
Efficient Subspace Fitting Algorithms for Diversely Polarized Arrays
}

\author{
A. SWINDLEHURST \\ Dept. of Elec. \& Comp. Engineering \\ Brigham Young University, Provo, UT 84602
}

\author{
M. VIBERG \\ Dept. of Electrical Engineering \\ Linköping University, S-58189 Linköping, SWEDEN
}

\begin{abstract}
Diversely polarized antenna arrays are widely used in $R F$ applications. The diversity of response provided by such arrays can greatly improve direction finding performance over arrays sensitive to only one polarization component. For d emitters, direct implementation of a multidimensional estimation algorithm (e.g., maximum likelihood) requires a search for $3 d$ parameters: $d$ directions of arrival (DOAs), and $2 d$ polarization parameters. In this paper, we derive a more efficent solution based on noise subspace fitting (NSF). The NSF algorithm is decoupled into a two-step procedure, where the DOAs are estimated first, and then the polarization parameters are obtained by solving a linear equation. The main advantage of this approach is that the search dimension is reduced by a factor of three. In addition, the algorithm can be shown to yield asymptotically minimum variance estimates provided no perfectly coherent signals are present.
\end{abstract}

\section{Introduction}

$\mathbf{M}$ OST ANTENNA ARRAYS used in radar and communications systems are sensitive to polarization differences between one received signal and another. Provided the array response for each polarization component can be separately calibrated, diversely polarized arrays are advantageous because they possess an extra degree of signal discrimination that can be used to improve detection and estimation performance. Despite this fact, relatively few algorithms for direction of arrival (DOA) estimation have been developed to take advantage of such arrays. Recent work in this area has focused on implementations for adaptive arrays [1], algorithms for exploiting multiple polarization dimensions (including both electrical and magnetic field components) [2], and performance analyses $[3,4]$. Extensions of the popular MUSIC and root-MUSIC algorithms to diversely polarized arrays have been considered in $[5,6,7]$, but similar ideas for multidimensional algorithms such as maximum likelihood have not been extensively studied. Notable exceptions include a recent algorithm that involves application of ESPRIT to a uniform linear array of

\footnotetext{
${ }^{1}$ This work was supported by the National Science Foundation under grant MIP-9110112, and by the IR\&D Program at ESL, Inc.
}

crossed dipoles [8], and a simulated annealing approach presented by Ziskind and Wax [9].

Conceptually, there is no real difficulty in extending multidimensional DOA estimation algorithms to the diversely polarized case. For example, one obvious method would be to simply augment the dimension of the parameter search to include the polarization parameters as well as the DOAs. However, this approach does not take advantage of the fact that the polarization parameters lie in a "linear parameter subspace" of the full parameter space [10]. In addition, such an approach triples the dimension of the parameter search, and requires one to somehow obtain initial estimates of the signal polarization before the DOAs can be consistently estimated.

The goal of this paper is to show how a more computationally efficient solution can be obtained for the noise subspace fitting (NSF) algorithm described in [11, 12] (also sometimes referred to as MODE). The proposed method exploits the special structure of the manifold for diversely polarized arrays to decouple the polarization estimation from that of the DOAs. In particular, the DOAs are obtained by a search procedure of standard order, while the polarization parameters are determined separately by solving a linear equation. If consistent initial estimates of the DOAs are available (e.g., from an initial application of this or some other algorithm, or from some prior estimates), our decoupled NSF approach can be shown to yield asymptotically efficient (minimum variance) parameter estimates.

\section{Background}

For an $m$-element array of sensors, we will define $\mathrm{a}(\theta) \in \mathbb{C}^{m}$ to be the array response for an emitter with direction-of-arrival $\theta$. The array manifold $\mathcal{A}=\{\mathbf{a}(\theta)$ : $\theta \in \Theta\}$ is assumed to be known, either analytically or via some calibration procedure. When sampled at some time instant $t$, the outputs of the $m$ array elements are stacked in a vector $\mathrm{x}(t) \in \mathbb{C}^{m}$. Assuming $d$ narrowband (co-channel) emitters are present, the array output $\mathbf{x}(t)$ may be written as

$$
\mathbf{x}(t)=\mathbf{A}(\boldsymbol{\theta}) \mathbf{s}(t)+\mathbf{n}(t),
$$


where $s(t) \in \mathbb{C}^{d}$ is the amplitude and phase of the signals at time $t, \mathbf{n}(t)$ is additive noise, and where

$$
\mathbf{A}(\boldsymbol{\theta})=\left[\mathbf{a}\left(\theta_{1}\right) \cdots \mathbf{a}\left(\theta_{d}\right)\right] \quad \boldsymbol{\theta}=\left[\theta_{1}, \cdots, \theta_{d}\right] .
$$

Assuming that the noise and signals are uncorrelated, and that the noise is spatially white, the covariance of the array data is given by

$$
\mathbf{R}=\mathcal{E}\left\{\mathbf{x}(t) \mathbf{x}^{*}(t)\right\}=\mathbf{A}(\boldsymbol{\theta}) \mathbf{S} \mathbf{A}^{*}(\boldsymbol{\theta})+\sigma^{2} \mathbf{I},
$$

where $(\cdot)^{*}$ denotes a complex conjugate transpose, $\mathbf{S}$ is the covariance matrix of the emitter signals, and $\sigma^{2}$ is the noise power at each sensor. In this paper, we will require that the emitter covariance $S$ be full rank $d$ (e.g., no perfect multipath is present). Using the model of (1), it is easily shown that the eigendecomposition of $\mathbf{R}$ has the following form:

$$
\mathbf{R}=\sum_{i=1}^{m} \lambda_{i} \mathbf{e}_{i} \mathbf{e}_{i}^{*}=\mathbf{E}_{s} \Lambda_{s} \mathbf{E}_{s}^{*}+\sigma^{2} \mathbf{E}_{n} \mathbf{E}_{n}^{*}
$$

where $\mathbf{E}_{s}=\left[\begin{array}{lll}\mathbf{e}_{1} & \cdots & \mathbf{e}_{d}\end{array}\right], \mathbf{E}_{n}=\left[\mathbf{e}_{d+1} \cdots \mathbf{e}_{m}\right]$, and $\lambda_{1} \geq$ $\cdots \geq \lambda_{d}>\lambda_{d+1}=\cdots=\lambda_{m}=\sigma^{2}$. The key observations to be made here are that the column space of $\mathbf{A}(\boldsymbol{\theta})$ and $\mathbf{E}_{s}$ coincide, and that $\mathbf{A}^{*}(\boldsymbol{\theta}) \mathbf{E}_{n}=0$. This fact suggests that, to determine $\theta$, we simply find the elements of $\mathcal{A}$ that are orthogonal to $\mathbf{E}_{n}$. Of course, since only a finite amount of data can be collected, the best we can do in practice is find the elements of $\mathcal{A}$ that are "closest" to being orthogonal to $\hat{\mathbf{E}}_{n}$, the eigenvectors of the sample average $\hat{\mathbf{R}}$.

\subsection{Diversely Polarized Array Data}

The response of a diversely polarized array can be decomposed into two parts, each due to a separate polarization component. Assume our $m$-element array has been calibrated using test signals with two different polarizations (e.g., horizontal and vertical). We thus have at our disposal two array manifolds, one for each of the polarization components:

$$
\mathcal{A}_{h}=\left\{\mathbf{a}_{h}(\theta): \theta \in \Theta\right\} \quad \mathcal{A}_{v}=\left\{\mathbf{a}_{v}(\theta): \theta \in \Theta\right\} .
$$

The most common type of polarization sensitive array measures a linear combination of the two polarization components, and is referred to in this paper as a superimposed polarization response (SPR) array. We will restrict our attention herein to SPR arrays, although an algorithm essentially identical to the one presented below can be derived to handle other cases (e.g., arrays that measure each polarization component separately) [13]. For SPR arrays, the total array response vector takes the form

$$
\mathbf{a}(\theta)=\mathbf{a}_{h}(\theta) \phi_{h}+\mathbf{a}_{v}(\theta) \phi_{v},
$$

where $\phi_{h}$ and $\phi_{v}$ are complex scalars representing the relative contribution of each polarization component. For $d$ emitter signals, we will let $\mathbf{A}_{h}(\boldsymbol{\theta})=\left[\mathbf{a}_{h}\left(\theta_{1}\right) \cdots \mathbf{a}_{h}\left(\theta_{d}\right)\right]$ and a similarly defined $\mathbf{A}_{v}(\boldsymbol{\theta})$ represent the steering matrices for each component, so that

$$
\mathbf{A}(\boldsymbol{\theta})=\mathbf{A}_{h}(\boldsymbol{\theta}) \boldsymbol{\Phi}_{h}+\mathbf{A}_{v}(\theta) \Phi_{v} \in \mathbb{C}^{m \times d},
$$

where $\boldsymbol{\Phi}_{h}=\operatorname{diag}\left\{\phi_{h 1}, \cdots, \phi_{h d}\right\}$ and $\boldsymbol{\Phi}_{v}$ is defined similarly. Note that any given diagonal element of $\boldsymbol{\Phi}_{h}$ could be zero, as long as the corresponding element in $\boldsymbol{\Phi}_{\boldsymbol{v}}$ is non-zero, and vice-versa. The diagonal elements of these matrices are also only unique to within a scale factor. The fact that $\mathbf{A}(\boldsymbol{\theta})$ is a linear function of the polarization parameters $\phi_{h i}$ and $\phi_{v i}$ makes it possible to find a more efficient solution than a brute force search for all parameters simultaneously.

\subsection{A MUSIC-Based Approach}

In the standard MUSIC algorithm, the steering vectors are searched one by one to find those values of $\theta$ for which $\mathbf{a}(\theta)$ is most orthogonal to $\hat{\mathbf{E}}_{n}$, according to the following measure:

$$
V_{M U}(\theta)=\frac{\mathbf{a}^{*}(\theta) \hat{\mathbf{E}}_{n} \hat{\mathbf{E}}_{n}^{*} \mathbf{a}(\theta)}{\mathbf{a}^{*}(\theta) \mathbf{a}(\theta)} .
$$

The various minima of $V_{M U}(\theta)$ are then taken to be the estimates of the DOAs. For a diversely polarized array, if we define

$$
\overline{\mathbf{A}}(\theta)=\left[\begin{array}{ll}
\mathbf{a}_{h}(\theta) & \mathbf{a}_{v}(\theta)
\end{array}\right] \quad \phi=\left[\begin{array}{c}
\phi_{h} \\
\phi_{v}
\end{array}\right],
$$

the MUSIC cost function becomes

$$
V_{M U}(\theta, \phi)=\frac{\phi^{*} \overline{\mathbf{A}}^{*}(\theta) \hat{\mathbf{E}}_{n} \hat{\mathbf{E}}_{n}^{*} \overline{\mathbf{A}}(\theta) \phi}{\phi^{*} \overline{\mathbf{A}}^{*}(\theta) \overline{\mathbf{A}}(\theta) \phi} .
$$

Since $\phi$ is unconstrained (except for $\phi \neq 0$ ), evaluating $V_{M U}(\theta, \phi)$ is equivalent to finding the following minimum generalized eigenvalue and eigenvector [6]:

$$
\overline{\mathbf{A}}^{*}(\theta) \hat{\mathbf{E}}_{n} \hat{\mathbf{E}}_{n}^{*} \overline{\mathbf{A}}(\theta) \mathbf{z}_{\min }=\lambda_{\min } \overline{\mathbf{A}}^{*}(\theta) \overline{\mathbf{A}}(\theta) \mathbf{z}_{\min }
$$

as a function of $\theta$. As proposed in [6], the DOA estimates can be found by viewing $\lambda_{\min }$ as a function of $\theta$, and searching for its minima. The polarization of the signal with DOA estimate $\hat{\theta}_{i}$ is then taken to be the eigenvector associated with $\lambda_{\min }\left(\hat{\theta}_{i}\right): \hat{\phi}_{i}=z_{\min }\left(\hat{\theta}_{i}\right)$.

\section{A Noise Subspace Fitting Approach}

In the noise subspace fitting (NSF) approach (sometimes referred to as MODE, for method of direction-ofarrival estimation) $[11,12]$, the DOAs are estimated to be those that minimize the following cost function:

$$
V_{N S F}(\theta)=\operatorname{Tr}\left(\mathbf{A}^{*}(\theta) \hat{\mathbf{E}}_{n} \hat{\mathbf{E}}_{n}^{*} \mathbf{A}(\theta) \mathbf{W}\right),
$$


where $\mathrm{W}=\mathrm{W}^{*}>0$ is a $d \times d$ weighting matrix. MUSIC is a special case of NSF for the choice $W=I$. For a non-diagonal $W$, the NSF algorithm must resort to a multidimensional search for the DOAs, and hence it is referred to as a multidimensional algorithm.

The choice of the weighting $\mathbf{W}$ is critical to algorithm performance. While any symmetric, positive definite $\mathbf{W}$ will yield consistent estimates, only a consistent estimate of the following weighting will yield statistically efficient (minimum variance) estimates:

$$
\mathbf{W}_{N S F}=\left(\mathbf{A}^{*}\left(\boldsymbol{\theta}_{0}\right) \mathbf{E}_{s}\left(\boldsymbol{\Lambda}_{s}-\sigma^{2} \mathbf{I}\right)^{-2} \Lambda_{s} \mathbf{E}_{s}^{*} \mathbf{A}\left(\theta_{0}\right)\right)^{-1}
$$

where $\theta_{0}$ is a vector containing the true DOAs.

In the case of a diversely polarized array, one might expect that NSF would require a search in $3 d$-dimensional space ( $d$ for the DOAs, and $2 d$ for the diagonal elements of $\Phi_{h}$ and $\Phi_{v}$ ). The goal of this section is to show that, as with MUSIC [6], a simpler solution is possible that decouples the DOA and polarization estimation. In particular, the method we present involves only a standard $d$-dimensional search for the DOAs, followed by a step in which the polarization parameters are solved for directly.

\subsection{Proposed Algorithm}

Since $\mathbf{A}=\mathbf{A}_{h} \boldsymbol{\Phi}_{h}+\mathbf{A}_{v} \boldsymbol{\Phi}_{v}$ (dropping explicit dependence on $\theta$ for simplicity), the NSF cost function has the form

$$
\begin{aligned}
V_{N S F}= & \operatorname{Tr}\left(\boldsymbol{\Phi}_{h}^{*} \mathbf{A}_{h}^{*} \hat{\mathbf{E}}_{n} \hat{\mathbf{E}}_{n}^{*} \mathbf{A}_{h} \boldsymbol{\Phi}_{h} \mathbf{W}\right)+\operatorname{Tr}\left(\boldsymbol{\Phi}_{h}^{*} \mathbf{A}_{h}^{*} \hat{\mathbf{E}}_{n} \hat{\mathbf{E}}_{n}^{*} \mathbf{A}_{v} \boldsymbol{\Phi}_{v} \mathbf{W}\right) \\
& +\operatorname{Tr}\left(\boldsymbol{\Phi}_{v}^{*} \mathbf{A}_{v}^{*} \hat{\mathbf{E}}_{n} \hat{\mathbf{E}}_{n}^{*} \mathbf{A}_{h} \boldsymbol{\Phi}_{h} \mathbf{W}\right)+\operatorname{Tr}\left(\boldsymbol{\Phi}_{v}^{*} \mathbf{A}_{v}^{*} \hat{\mathbf{E}}_{n} \hat{\mathbf{E}}_{n}^{*} \mathbf{A}_{v} \boldsymbol{\Phi}_{v} \mathbf{W}\right)
\end{aligned}
$$

It is easily shown that, for a diagonal matrix $\Phi$ and arbitrary square matrices $\mathbf{X}$ and $\mathbf{Y}$, the relation

$$
\operatorname{Tr}\left(\boldsymbol{\Phi}^{*} \mathbf{X} \boldsymbol{\Phi} \mathbf{Y}\right)=\phi^{*}\left(\mathbf{X} \odot \mathbf{Y}^{T}\right) \boldsymbol{\phi}
$$

holds, where $\phi$ is the vector formed from the diagonal elements of $\boldsymbol{\Phi}$, and $\odot$ represents a Hadamard (element by element) product. Using (9), the NSF criterion may be rewritten as

$$
V_{N S F}(\theta, \phi)=\phi^{*} \mathbf{M}_{s}(\theta) \phi,
$$

where $\phi=\left[\begin{array}{ll}\phi_{h}^{T} & \phi_{v}^{T}\end{array}\right]^{T}, \phi_{h}$ and $\phi_{v}$ represent the diagonal elements of $\boldsymbol{\Phi}_{h}$ and $\Phi_{v}$, respectively, and

$$
\mathbf{M}_{s}(\theta)=\left[\begin{array}{ll}
\left(\mathbf{A}_{h}^{*} \hat{\mathbf{E}}_{n} \hat{\mathbf{E}}_{n}^{*} \mathbf{A}_{h}\right) \odot \mathbf{W}^{T} & \left(\mathbf{A}_{h}^{*} \hat{\mathbf{E}}_{n} \hat{\mathbf{E}}_{n}^{*} \mathbf{A}_{v}\right) \odot \mathbf{W}^{T} \\
\left(\mathbf{A}_{v}^{*} \hat{\mathbf{E}}_{n} \hat{\mathbf{E}}_{n}^{*} \mathbf{A}_{h}\right) \odot \mathbf{W}^{T} & \left(\mathbf{A}_{v}^{*} \hat{\mathbf{E}}_{n} \hat{\mathbf{E}}_{n}^{*} \mathbf{A}_{v}\right) \odot \mathbf{W}^{T}
\end{array}\right] .
$$

Note that in the formulation of NSF in (10), $\phi$ contains the $2 d$ complex polarization parameters, while $\mathbf{M}_{s}(\theta)$ is a function of the DOAs only Because of the form of the cost function in (10), it is natural to suppose that minimizing $V_{N S F}$ is equivalent to finding the $\theta$ which minimizes the smallest eigenvalue of $M_{s}(\theta)$, and then estimating the polarization parameters from the eigenvector associated with the minimum eigenvalue. However, this will not be the case since constraints on $\phi$ (other than $\phi^{*} \phi=1$ ) are necessary to guarantee identifiability.

Since the polarization can be scaled arbitrarily, there are actually only $d$ "free" complex polarization parameters, though $\phi$ is a $2 d$-vector. One possible uniqueness constraint is to force $\boldsymbol{\Phi}_{v}=I$ and solve only for $\phi_{h}$. A drawback to this approach is that it implicitly assumes that all of the signals have a non-zero (vertical) polarization. While the problem could easily be reformulated to force $\boldsymbol{\Phi}_{h}=\mathrm{I}$, it is desirable to develop a more general approach that handles situations involving arbitrary signals. One such approach is to use the following constraint:

$$
\operatorname{Re}\left\{\phi_{h}+\phi_{v}\right\}=\operatorname{Im}\left\{\phi_{h}+\phi_{v}\right\}=\mathbf{e},
$$

where $\operatorname{Re}\{\cdot\}$ and $\operatorname{Im}\{\cdot\}$ denote real and imaginary parts, respectively; and where $e$ is a vector of all ones. Using this constraint, the NSF minimization becomes

$$
\{\hat{\theta}, \hat{\phi}\}=\arg \min _{\theta, \phi} \phi^{*} \mathbf{M}_{s}(\theta) \phi \quad \text { subject to } \mathbf{B}^{*} \phi=\mathbf{e}
$$

where

$$
\mathbf{B}=\frac{1}{1-j}\left[\begin{array}{l}
\mathbf{I} \\
\mathbf{I}
\end{array}\right]
$$

To solve (12), we incorporate the constraint into the cost function by noting that all $\phi$ that satisfy $\mathbf{B}^{*} \phi=e$ can be parameterized as

$$
\phi=\mathbf{B} \mathbf{e}+\mathbf{B}^{\perp} \boldsymbol{\psi},
$$

where $\mathbf{B}^{*} \mathbf{B}^{\perp}=0$ and $\boldsymbol{\psi}$ is an arbitrary $d$-vector. The matrix $\mathbf{B}^{\perp}$ is also otherwise arbitary; for example, one could choose

$$
\mathbf{B}^{\perp}=\frac{1}{1+j}\left[\begin{array}{r}
\mathbf{I} \\
-\mathbf{I}
\end{array}\right] \text {. }
$$

Writing the cost function in terms of e and $\psi$, and setting $\partial V_{N S F} / \partial \psi=0$ allows us to separate out the estimation of $\psi$ :

$$
\hat{\psi}=-\left[\mathbf{B}^{\perp *} \mathbf{M}_{s}(\theta) \mathbf{B}^{\perp}\right]^{-1} \mathbf{B}^{\perp *} \mathbf{M}_{s}(\theta) \mathbf{B e},
$$

from which we obtain an estimate of $\phi$ :

$$
\hat{\boldsymbol{\phi}}=\left(\mathbf{I}-\mathbf{B}^{\perp}\left[\mathbf{B}^{\perp *} \mathbf{M}_{s}(\theta) \mathbf{B}^{\perp}\right]^{-1} \mathbf{B}^{\perp *} \mathbf{M}_{s}(\theta)\right) \mathbf{B e} .
$$

Substituting this solution into (12) yields the following cost function which depends only on $\boldsymbol{\theta}$ :

$$
\mathrm{e}^{T}\left(\mathrm{M}_{s}-\mathrm{M}_{s} \mathbf{B}^{\perp}\left[\mathrm{B}^{\perp *} \mathrm{M}_{s} \mathbf{B}^{\perp}\right]^{-1} \mathbf{B}^{\perp *} \mathrm{M}_{s}\right) \mathrm{e},
$$

where e is now of length $2 d$. This constrained algorithm is implemented as follows:

1. Find the $\theta$ that minimizes (15) (i.e., the sum of the elements of the matrix in the middle).

2. Solve for $\hat{\phi}$ using $\hat{\theta}$ in equation (14). 
The discussion above demonstrates how to guarantee the identifiability of the DOAs $\theta$ by properly constraining $\phi$. In [13], it is shown that the specific choice of the constraint does not affect the asymptotic performance of the algorithm, as long as $\phi$ remains unique. In addition to the constraints on $\phi$, certain conditions on the array must also be met to guarantee the identifiability of $\phi$. For SPR arrays, it turns out that the following conditions on the array are sufficient: $\operatorname{rank}\left(\mathbf{A}_{h}\right)=$ $\operatorname{rank}\left(\mathbf{A}_{v}\right)=d$, and $\operatorname{rank}\left(\left[\begin{array}{ll}\mathbf{a}_{h}(\theta) & \mathbf{a}_{v}(\theta)\end{array}\right]\right)=2 \forall \theta$

\section{A Simulation Example}

To compare the performance of the above NSF methods with the MUSIC-based approach of [6], a simple simulation study was conducted for the SPR case. A 10 element uniform linear array (ULA) was assumed for the $\mathcal{A}_{h}$ manifold, while $\mathcal{A}_{v}$ was generated by slightly perturbing the element positions of a 10 element ULA (to guarantee diverse polarization). Two $10 \mathrm{~dB}$ emitters were simulated at $0^{\circ}$ and $10^{\circ}$ relative to broadside, with polarization defined by $\phi_{h 1}=\phi_{h 2}=1$, and $\phi_{v 1}=e^{j 6 \pi / 5}$, $\phi_{v 2}=e^{j \pi / 10}$, respectively. All sensors were assumed to have unity gain, so the effective SNR for each source was between 12 and $13 \mathrm{~dB}$. A total of 100 snapshots were taken from the array in 250 independent trials, and the RMS error of the DOA estimates was calculated for MUSIC and NSF.

The results are plotted in Figure 1 for the emitter at $10^{\circ}$. In the figure, constraint 1 refers to the NSF algorithm using (11), while constraint 2 indicates the NSF algorithm assumed $\phi_{h}=e$. When the signals are $50 \%$ correlated or less, we see that all three methods give similar results. At higher correlation levels, the relative performance of NSF becomes increasingly better than that of MUSIC.

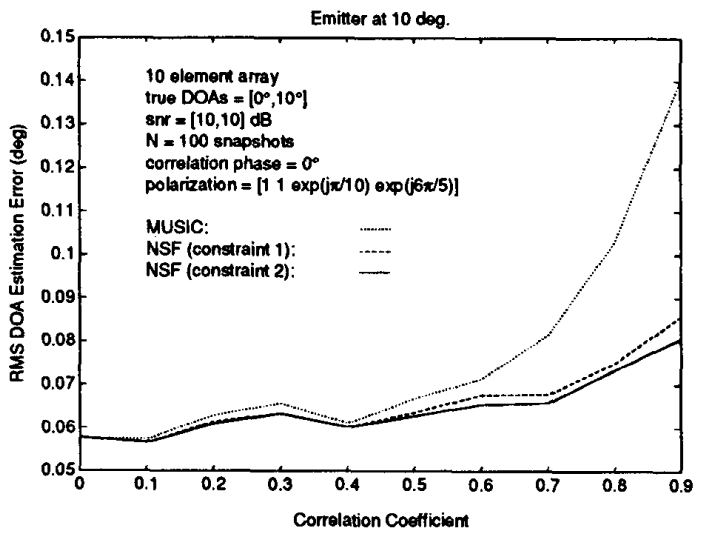

Figure 1: Comparison of MUSIC and NSF DOA Estimates

\section{Conclusions}

We have presented a statistically efficient algorithm for DOA estimation using diversely polarized arrays. The proposed technique requires a multidimensional search over the DOA parameters only, after which the polarization parameters can be calculated explicitly. A comparison with a MUSIC-based approach revealed that our method may give significantly improved performance, particularly in scenarios involving highly correlated signals.

\section{References}

[1] R. T. Compton, Jr., "The Tripole Antenna: An Adaptive Array with Full Polarization Flexibility", IEEE Trans. on Antennas and Propagation, AP-29:944-952, November 1981.

[2] A. Nehorai and E. Paldi, "Vector Sensor Processing for Electromagnetic Source Localization", In Proc. $25^{\text {th }}$ Asilomar Conference on Signals, Systems, and Computers, pages 566572, Asilomar, CA., November 1991.

[3] A. J. Weiss and B. Friedlander, "Performance Analysis of Diversely Polarized Antenna Arrays", IEEE Trans. on Sig. Proc., SP-39(7):1589-1603, July 1991.

[4] A. Nehorai and E. Paldi, "Vector Sensor Processing for Electromagnetic Source Localization", Technical Report 9209, Yale University, May 1992.

[5] R. Schmidt, A Signal Subspace Approach to Multiple Emitter Location and Spectral Estimation, PhD thesis, Stanford University, 1981.

[6] E. R. Ferrara and T. M. Parks, "Direction Finding with an Array of Antennas Having Diverse Polarizations", IEEE Trans. on Antennas and Propagation, AP-31(2):231-236, March 1983.

[7] B. Friedlander and A. J. Weiss, "A Direction Finding Algorithm for Diversely Polarized Arrays", Digital Signal Processing - A Review Journal, 2(3):123-134, July 1992.

[8] J. Li and R. Compton, Jr., "Angle and Polarization Estjmation Using ESPRIT with a Polarization Sensitive Array", IEEE Trans. on Antennas and Propagation, AP-39:13761383, September 1991.

[9] I. Ziskind and M. Wax, "Maximum Likelihood Localization of Diversely Polarized Sources by Simulated Annealing", IEEE Trans. on Antennas and Propagation, AP-38:11111114, July 1990.

[10] R. Schmidt, "Multilinear Array Manifold Interpolation", IEEE Trans, on Sig. Proc,, 40(4):857-866, April 1992.

[11] H. Clergeot, S. Tressens, and A. Ouamri, "Performance of High-Resolution Frequencies Estimation Methods Compared to the Cramér-Rao Bounds", IEEE Trans. on ASSP, 37(11):1703-1720, Nov. 1989.

[12] P. Stoica and K. Sharman, "Maximum Likelihood Methods for Direction-of-Arrival Estimation", IEEE Trans. on ASSP, 38(7):1132-1143, July 1990.

[13] A. Swindlehurst and M. Viberg, "Subspace Fitting with Diversely Polarized Arrays", submitted to IEEE Trans. on Ant. and Prop., January 1993. 